\title{
Possible Signature of Sausage Waves in Photospheric Bright Points
}

\author{
Yuhang $\mathrm{Gao}^{1} \bullet \bullet \cdot$ Fuyu $\mathrm{Li}^{2} \bullet \bullet \cdot \mathrm{Bo} \mathrm{Li}^{3} \bullet \bullet \cdot$ \\ Wenda $\mathrm{Cao}^{4} \bullet \bullet \cdot$ Yongliang Song $^{5} \bullet \bullet \cdot$ \\ Hui Tian ${ }^{1,5} \bullet \bullet \cdot$ Mingzhe Guo ${ }^{3} \bullet \bullet$
}

(C) Springer $\bullet \bullet \bullet \bullet$

\begin{abstract}
Sausage waves have been frequently reported in solar magnetic structures such as sunspots, pores, and coronal loops. However, they have not been unambiguously identified in photospheric bright points (BPs). Using high-resolution TiO image sequences obtained with the Goode Solar Telescope at the Big Bear Solar Observatory, we analyzed four isolated BPs. It was found that their area and average intensity oscillate for several cycles in an in-phase fashion. The oscillation periods range from 100 to 200 seconds. We interpreted the phase relation as a signature of sausage waves, particularly slow waves, after discussing the sausage wave theory and the opacity effect.
\end{abstract}

Keywords: Photospheric Bright Points; Waves, Magnetohydrodynamic; Magnetic Flux Tube; Opacity Effect

\footnotetext{
$\triangle$ F. Li

lifuyu@ioe.ac.cn

1 School of Earth and Space Sciences, Peking University, Beijing 100871, People's Republic of China

2 Institute of Optics and Electronics, Chinese Academy of Sciences, Chengdu, Sichuan, 610209, People's Republic of China

3 Shandong Provincial Key Laboratory of Optical Astronomy and Solar-Terrestrial Environment, Institute of Space Sciences, Shandong University, Weihai, Shandong 264209, People's Republic of China

4 Big Bear Solar Observatory, New Jersey Institute of Technology, Big Bear City, CA 92314, USA

5 Key Laboratory of Solar Activity, National Astronomical Observatories, Chinese Academy of Sciences, Beijing 100012, People's Republic of China
} 


\section{Introduction}

Photospheric bright points (BPs) are dynamic, small-scale, bright structures in the solar photosphere, usually appearing in dark intergranular lanes. They are associated with magnetic elements in magnetograms and have a magnetic-field strength of about $1 \mathrm{kG}$. BPs are typically circular (Berger et al., 1995; Bovelet and Wiehr, 2003), while some of them have elongated shapes (Kuckein, 2019). Their equivalent diameter is about $\mathbf{1 0 0 -} \mathbf{3 0 0} \mathbf{~ k m}$, and their lifetime is about 90 seconds - 10 minutes (Berger and Title, 1996; Utz et al., 2010; Keys et al., 2011, 2014). In addition, BPs usually have a brightness contrast of $\mathbf{0 . 8} \mathbf{- 1 . 8}$ relative to the mean intensity of the photosphere (Sánchez Almeida et al., 2004).

BPs were first discovered in G-band observations of the photosphere in the 1970s (Dunn and Zirker, 1973), and later Mehltretter (1974) found that they represent magnetic-flux concentrations by comparing Ca II $\mathrm{K}$ images with magnetograms. BPs are therefore also called magnetic bright points, and they are considered as the photospheric footpoints of magnetic flux tubes (e.g. Berger et al., 1998; De Pontieu, 2002). A process named convective collapse is believed to be their formation mechanism (Spruit, 1979), which has received some observational support (e.g. Solanki et al., 1996; Bellot Rubio et al., 2001; Nagata et al., 2008).

Since BPs can be regarded as tracers of photospheric magnetic flux tubes, many studies have suggested that BPs may host a variety of MHD waves (Martínez González et al., 2011; Jess et al., 2012a; Mumford, Fedun, and Erdélyi, 2015; Jafarzadeh et al., 2017). Given that the dissipation of MHD waves is among the most promising mechanisms for heating the solar corona, it is of great significance to study MHD waves associated with BPs or photospheric magnetic elements. So far, possible observational manifestations of kink waves (Stangalini, Berrilli, and Consolini, 2013; Stangalini, Giannattasio, and Jafarzadeh, 2015) and Alfvén waves (Jess et al., 2009) have already been found in BPs (or magnetic elements). However, sausage waves have not been unambiguously identified in them.

Sausage waves (or sausage modes) in a flux tube are characterised by a sausage-like boundary of the tube, first defined by Defouw (1976). A detailed discussion of this wave mode is given in Section 4.1. There are many studies focusing on theoretical understanding and seismological applications of sausage modes (e.g. Chen et al., 2015; Guo et al., 2016; see also the review by Li et al., 2020). In observations, sausage modes in the photosphere have been widely studied in magnetic pores and sunspots (e.g. Dorotovič, Erdélyi, and Karlovský, 2008; Morton et al., 2011; Grant et al., 2015; Freij et al., 2016). Also, Morton et al. (2012) found evidence for the simultaneous existence of sausage and kink modes in fine magnetic structures in the chromosphere. In the corona, shortperiod, quasi-periodic pulsations (QPPs) in flares are also generally interpreted as sausage waves (e.g. Nakariakov, Melnikov, and Reznikova, 2003; Van Doorsselaere et al., 2011; Tian et al., 2016; see also the review by Zimovets et al., 2021).

In this article, we report the existence of in-phase oscillations of area and average intensity in BPs using high-resolution TiO images for the first time. Our findings can be regarded as possible evidence of sausage modes in photospheric 


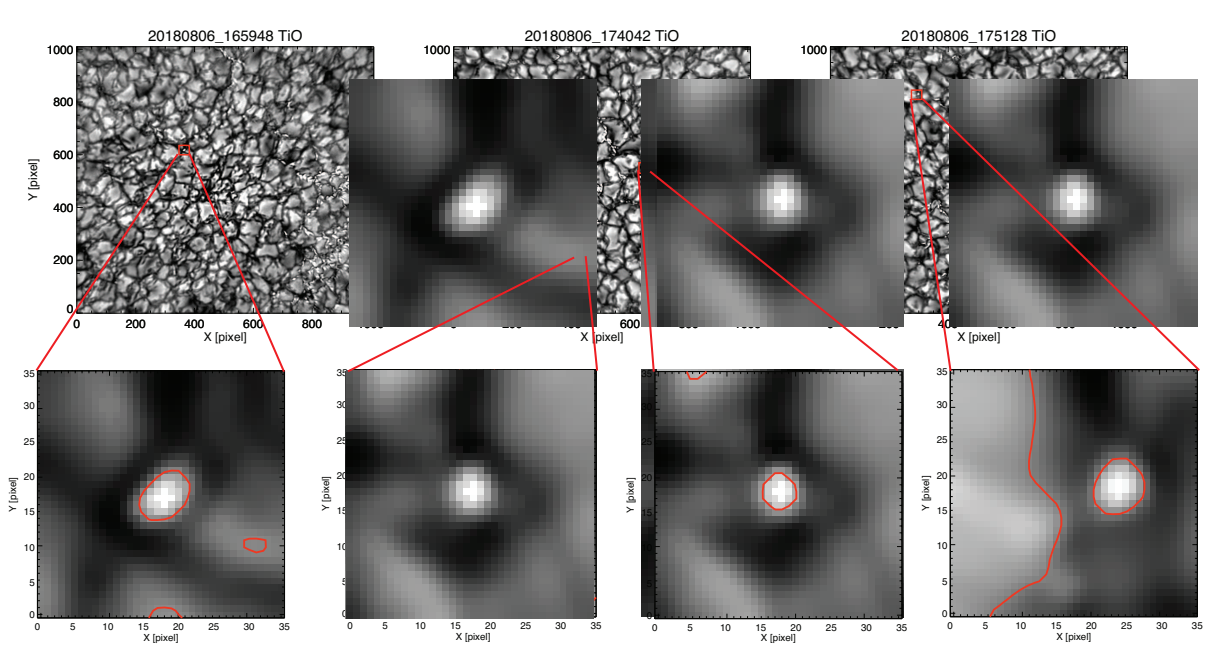

Figure 1. TiO images with BPs marked by boxes. The images below show an enlarged view of the red box regions, where BPs are identified and tracked with red contours.

flux tubes. In fact, magnetoacoustic waves characterised by intensity or line-ofsight (LOS) velocity oscillations have been found in BPs before (Jess et al., 2012a,b; Stangalini et al., 2013; Jafarzadeh et al., 2017). But these preivious studies could not tell whether these waves were sausage waves, because either they did not consider BP areas or the area variations were too weak. Martínez González et al. (2011) studied four magnetic patches in circular polarization images obtained by the Imaging Magnetograph eXperiment (IMaX) on board Sunrise, and they found that the iso-flux area oscillates with time. However, the time-varying periods suggested that the oscillations may be caused by granular motions rather than a chacteristic oscillation mode. Fujimura and Tsuneta (2009) also focused on oscillations of intergranular magnetic structures (IMSs) in a magnetogram and interpreted these oscillations as sausage and/or kink waves. But the lifetimes of these IMSs are all greater than one hour, which is much longer than the typical lifetime of BPs. Kolotkov et al. (2017) also detected long-period (80-230 minutes) quasi-periodic oscillations of the average magnetic field and total area of a small magnetic structure. More recently, Keys et al. (2020) studied the rapid variations of magnetic fields in BPs and believed that such changes are consistent with the behavior of MHD waves, but they did not discuss in detail the changes of intensity.

This article is organised as follows. In Section 2, we describe our observation and data analysis methods, and the key results are presented in Section 3. We discuss the wave-mode identification in Section 4 before giving the final conclusion and some remarks in Section 5. 
Table 1. Observational details of the BPs. The area is averaged over the time, and C.C. represents the correlation coeffecient of average intensity and area.

\begin{tabular}{cccccc}
\hline & Date & Time [UT] & Location & Area $\left[\mathbf{M m}^{2}\right]$ & C.C. \\
\hline BP1 & 06 Aug. 2018 & $16: 54: 18-17: 03: 18$ & $\left(501^{\prime \prime},-222^{\prime \prime}\right)$ & 0.015 & 0.71 \\
BP2 & 06 Aug. 2018 & $17: 38: 12-17: 46: 28$ & $\left(-260^{\prime \prime}, 2^{\prime \prime}\right)$ & 0.017 & 0.92 \\
BP3 & 06 Aug. 2018 & $17: 33: 12-17: 41: 27$ & $\left(-260^{\prime \prime}, 2^{\prime \prime}\right)$ & 0.013 & 0.69 \\
BP4 & 06 Aug. 2018 & $17: 47: 43-17: 53: 43$ & $\left(-260^{\prime \prime}, 2^{\prime \prime}\right)$ & 0.015 & 0.89 \\
\hline
\end{tabular}

\section{Observations and Data Analysis}

The data used in this study were acquired with the 1.6-meter Goode Solar Telescope (GST; Cao et al., 2010) at the Big Bear Solar Observatory (BBSO). We used the Broadband Filter Imager (BFI) installed on the GST to observe the TiO band ( $7057 \AA$ ) from 16:28 to 17:59 UT on 6 August 2018, with a cadence of 15 seconds. The observed regions include the active region NOAA 12717 at $\left(501^{\prime \prime},-222^{\prime \prime}\right)$ from 16:28 to $17: 33$ and the western lobe of a coronal hole at $\left(-260^{\prime \prime}, 2^{\prime \prime}\right)$ from $17: 33$ to $17: 59$.

The bandwidth of the filter is $10 \AA$. The field of view (FOV) of the TiO data was $77^{\prime \prime} \times 77^{\prime \prime}$ and the pixel size was $0.034^{\prime \prime}$. During the observation, the seeing condition was better than $3^{\prime \prime}$, which is good for the identification of BPs. Speckle reconstruction and a high-order adaptive optics (AO) system were used to reach the spatial-resolution limit. The data were also destretched to remove the residual atmospheric-seeing effects.

We first selected isolated BPs in the FOV by eye. Liu et al. (2018) and Kuckein (2019) divided BPs into isolated ones, which are individual, and non-isolated ones, which display a splitting or merging behavior in observations. Obviously, it is more suitable to use isolated BPs to search for MHD waves. Because for non-isolated BPs, their splitting and merging behaviors may affect our identification of waves. For the selected BPs, we used contours with a fixed intensity threshold level to identify and track them. Then we calculated the area and average intensity of these BPs at each time and examined if there is any oscillation signal. Finally, four of the BPs are found to be well isolated and there is also possible evidence of sausage waves inside. Their positions and shapes are shown in Figure 1. We named them BP1 to BP4. When calculating their average intensity and area, we carefully removed the unrelated contours nearby (we can see them around BP1, BP2, and BP4 in Figure 1) by manually zooming on the region of interest for every frame. We summarize the properties of the four BPs in Table 1.

\section{Result}

Figure 2 presents the time series of the area and average intensity of these four BPs, and we can see obvious oscillations for two to four cycles. It can also 

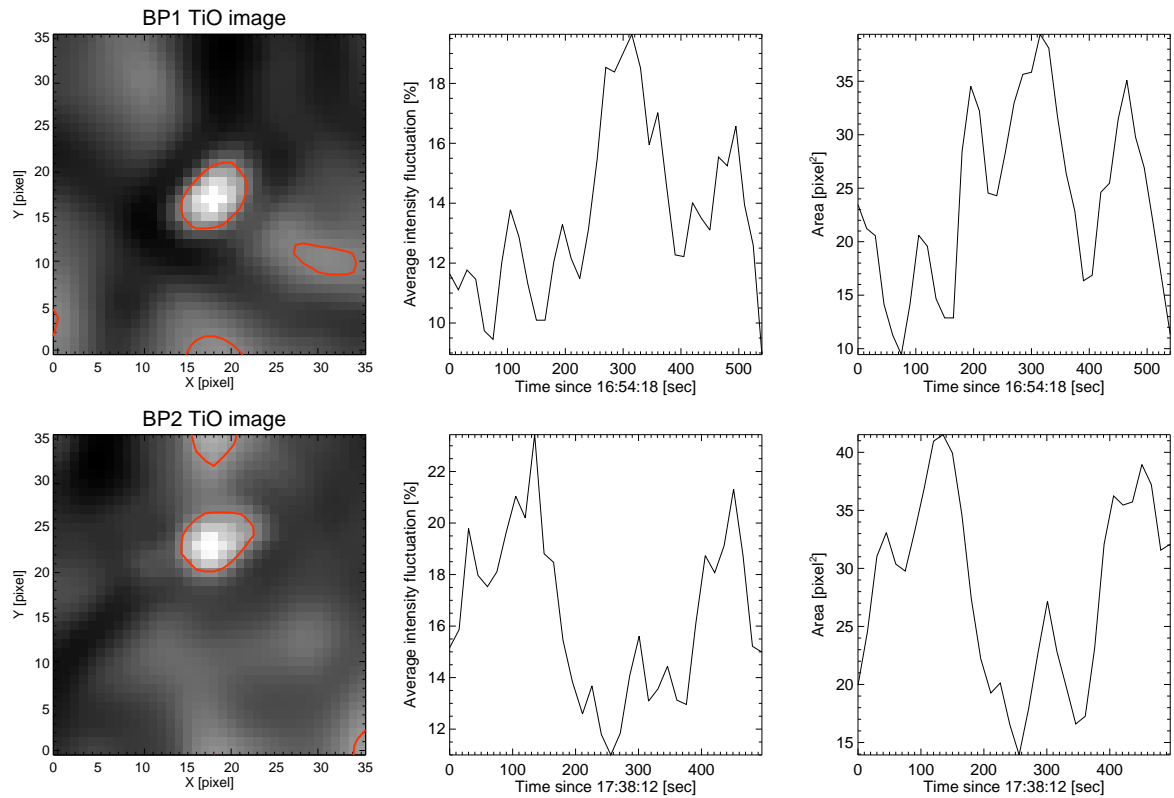

BP3 TiO image
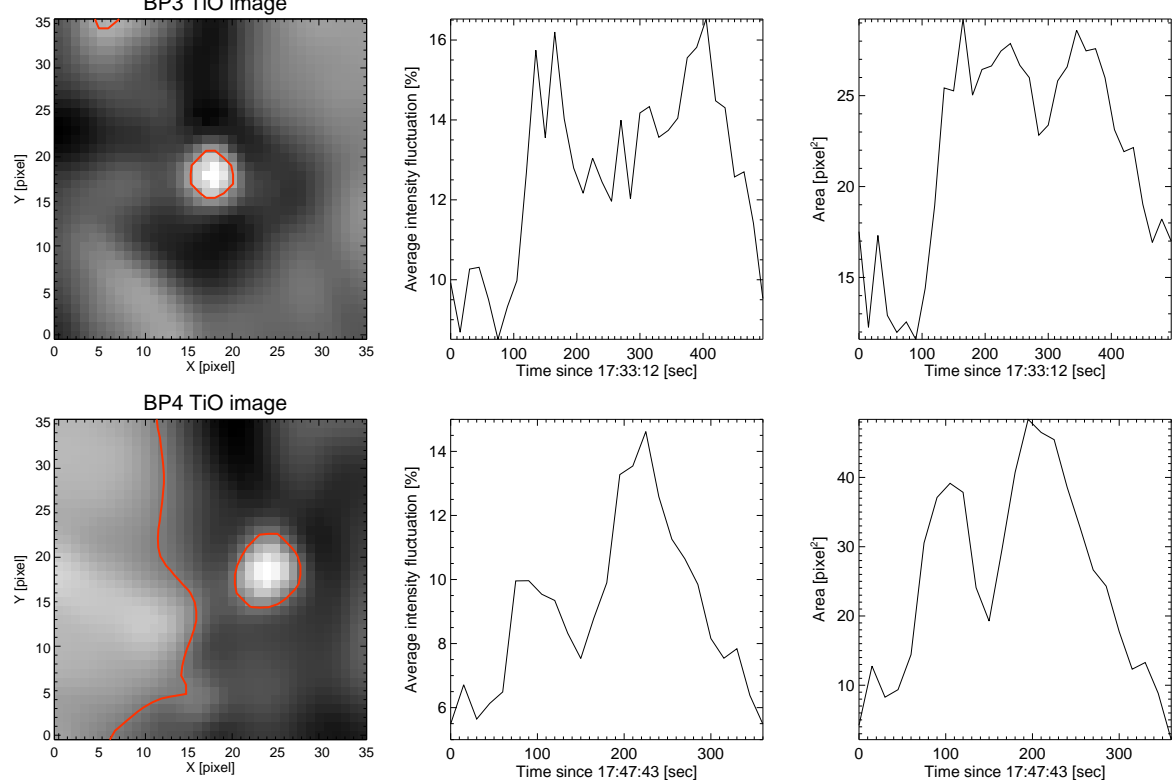

Figure 2. Time series of average intensity and area of the four BPs. Each row corresponds to one $\mathrm{BP}$, and the right two columns show the temporal variations of the average intensity and area, respectively. Here we use the intensity fluctuation percentage with respect to the mean value of the backgroud. (Animation of this figure is available in the Supplementary Materials.) 


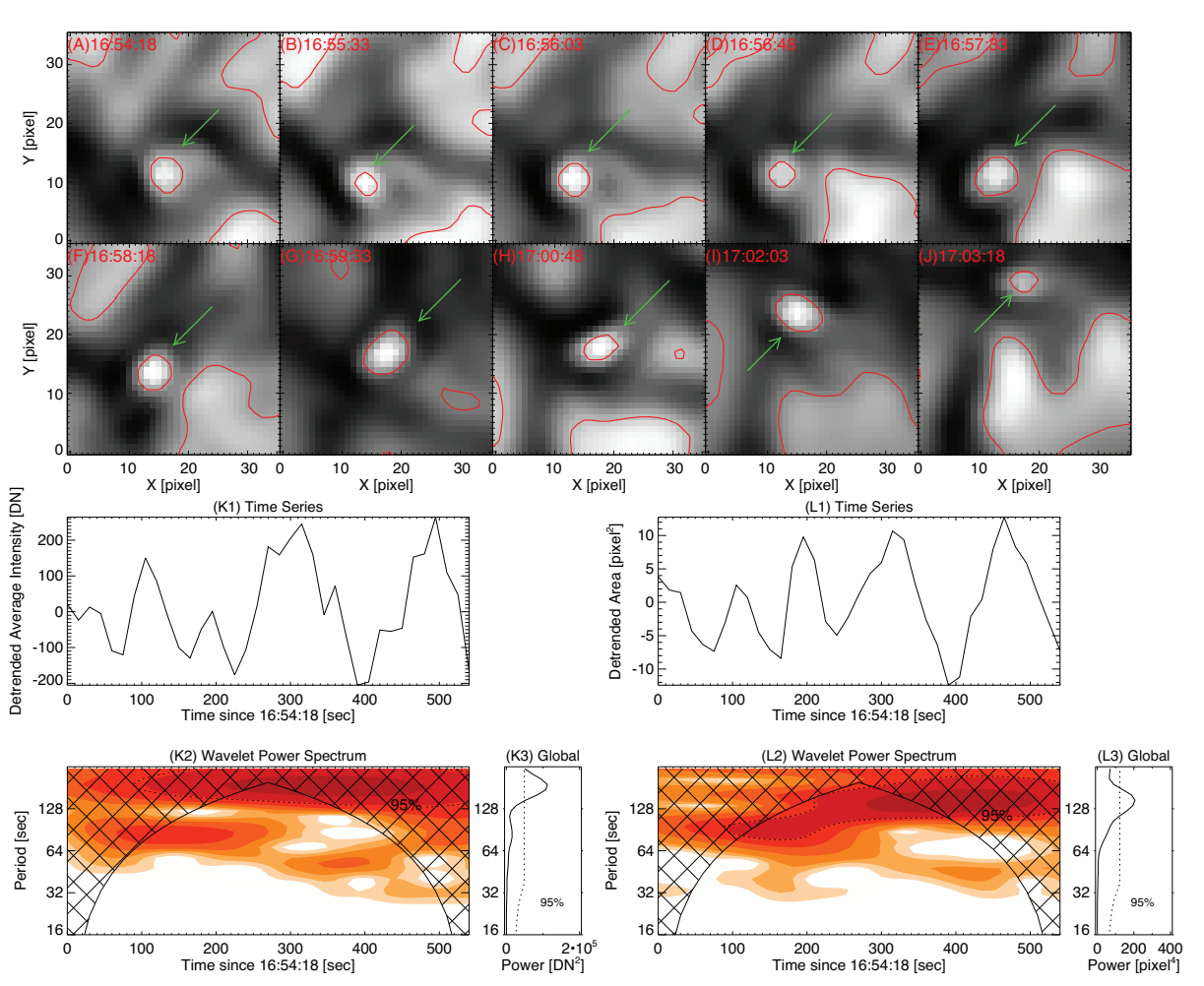

Figure 3. $(\mathbf{A}-\mathbf{J})$ : snapshots of BP1 marked by green arrows at ten different time. Approximately, every three adjacent panels correspond to an oscillation cycle. (K): wavelet analysis results of detrended time series (shown in Panel K1) of average intensity. The results include the wavelet power spetrum (Panel K2) and the global power spectrum (Panel K3). Darker colors represent stronger powers. The dashed lines indicate a significance level of $95 \%$. (L): same as Panel $\mathrm{K}$ but for the detrended time series of area.

be noticed that for each BP, the crests and troughs for these two features appear at nearly the same time, which suggests that the oscillations of area and average intensity are in phase. Among these BPs, BP1 has the most oscillation cycles, with four clear crests in the time series of area, respectively at 105, 195, 315, and 465 seconds. These instants correspond well to crests in the curve of average intensity. In addition, there is also a good correspondence between moments of each trough. Figure 3 gives ten snapshots of BP1 in Panels $\mathbf{A}-\mathbf{J}$, each corresponding to a moment of wave crest or trough. We can clearly see that the BP's area oscillates with time.

Then we detrended the time series of average intensity and area by subtracting a three-minute running average before performing wavelet analysis. The detrended series and wavelet analysis results are shown in Panels $\mathrm{K}$ and L of Figure 3. The peak global wavelet powers for the average intensity and area are at around 150 seconds, which could be seen as the oscillation period. For the other BPs, the periods can be estimated to be 100-200 seconds. The period range we found here are comparable to the results in some previous studies, which interpreted the oscillations they found in BPs as magnetoacoustic 

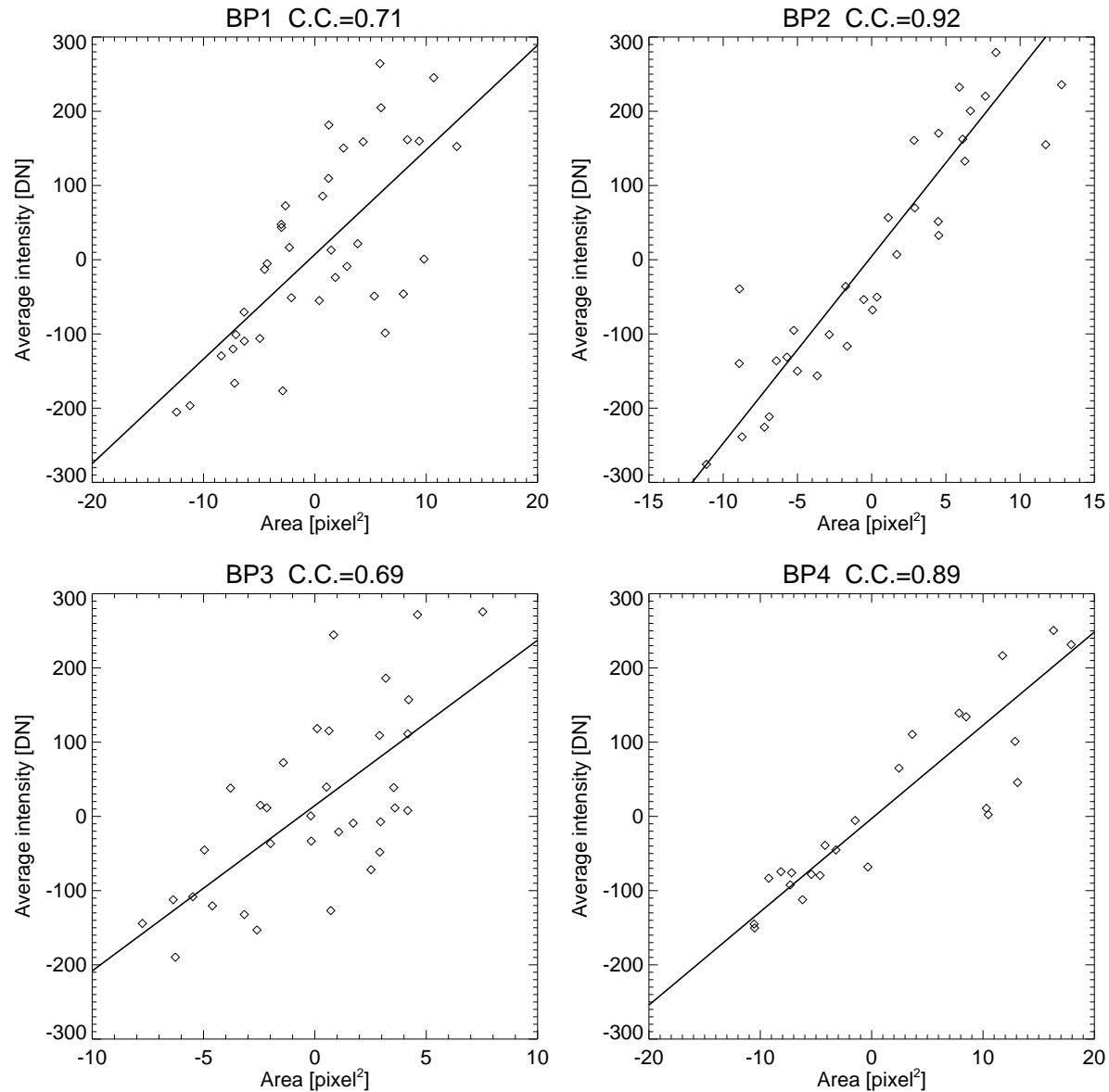

Figure 4. Scatter plots of the relationship between average intensity and area for the four BPs, with solid lines representing linear fits. Note that the average intensity and area are both detrended.

waves (Jess et al., 2012a,b). We note that oscillations with longer periods above four minutes were detected in small magnetic structures before (Fujimura and Tsuneta, 2009; Martínez González et al., 2011; Kolotkov et al., 2017).

In order to further confirm the in-phase relation between oscillations of average intensity and area, we drew scatter plots of relationship between them, and calculated the correlation coefficients, as shown in Figure 4. It can be seen that the correlation coefficients are all above 0.68. For BP2 and BP4, the coefficients are around 0.9 , showing a good positive correlation. Meanwhile, we also performed a lagged cross-correlation (LCC) analysis. From Figure 5, we can see that the coeffients for all BPs reach the peak when time lag is zero. All the results confirm the in-phase relation between average intensity and area.

A possible question is whether the correlation or the in-phase relation is caused by our analysis method, i.e. using contours with a fixed intensity threshold to identify BPs and calculate their parameters. If we consider the total intensity 

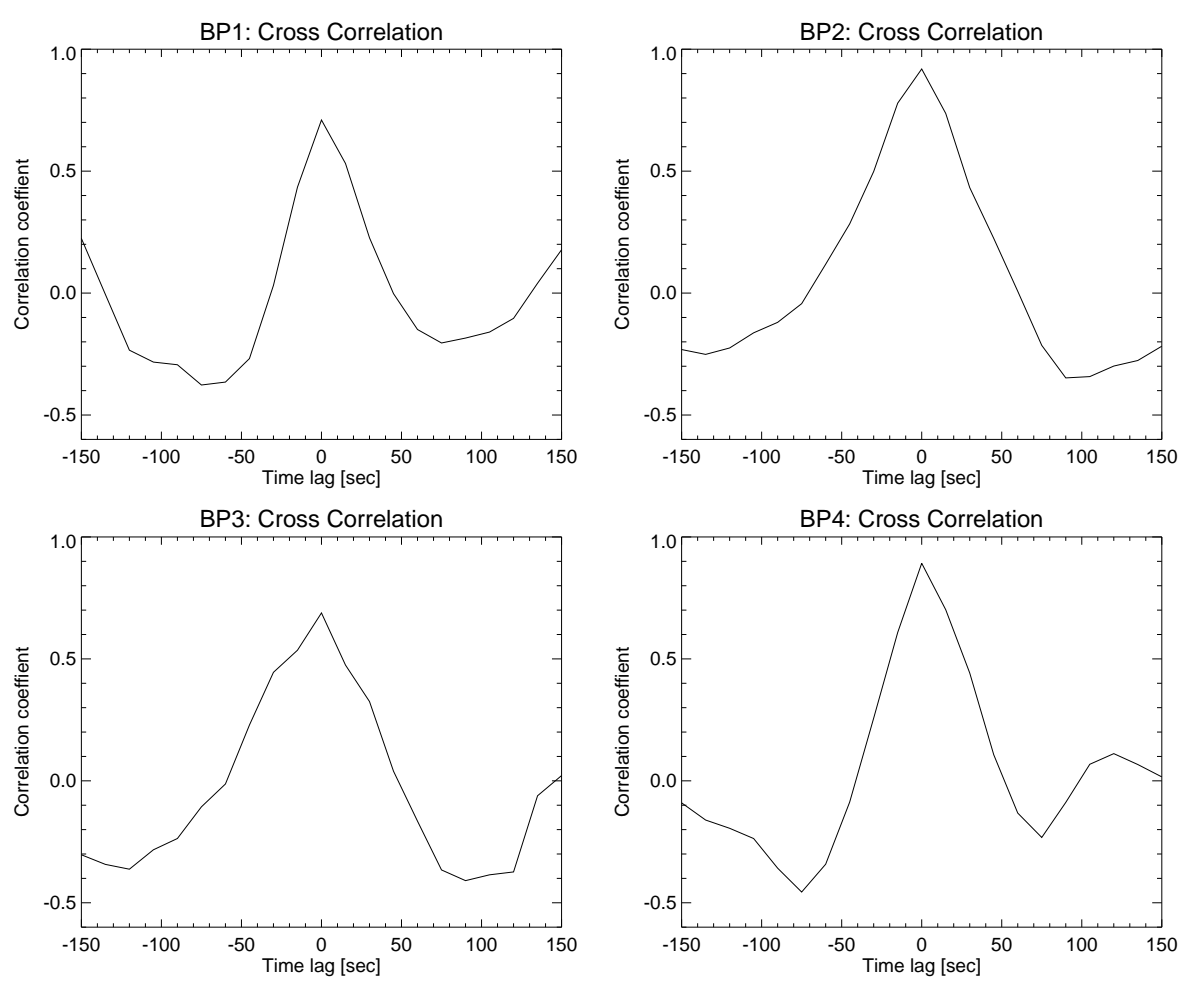

Figure 5. Lagged cross-correlations between average intensity and area. The time lag is between the detrended time series of area and average intensity.

rather than the average intensity in the contours, we might tend to obtain an in-phase relation, since it seems that the total intensity will always increase (or decrease) along with the increase (or decrease) of the area. So here we chose to use time series of the average intensity. Additionally, we studied for more than four isolated BPs with the same method, and we found that there are cases without such an oscillation pattern. In conclusion, the in-phase relation is not a result of our data-processing method, but more likely associated with some characteristic oscillation modes.

We will show in the next section that the observed in-phase oscillations might be explained as sausage waves in BPs.

\section{Discussion}

\subsection{Sausage Mode Waves in a Flux Tube}

In the solar atmosphere, magnetic flux tubes are formed with magnetic field lines clumping into tight bundles. They can be regarded as channels guiding MHD waves. A flux tube is usually assumed as a straight cylinder described by cylindrical coordinates $(r, \phi, z)$. The tube boundary can oscillate in different 
geometrical patterns, forming various wave modes including sausage modes, kink modes, fluting modes, and torsional (Alfvén) modes (Roberts, 2019). For sausage modes, the axis of the tube is motionless, while the cross-section area fluctuates with time, resulting in a sausage-like axisymmetric perturbation of the tube boundary. So sausage waves are axisymmetric waves, belonging to magnetoacoustic waves. According to the relationship between phase speed $c_{\mathrm{p}}$ and sound speed $c_{\mathrm{s}}$ in the tube, we can divide the sausage wave into a fast mode and slow mode. The former has a faster phase speed than the sound speed, while the latter has a slower phase speed. Assumed here and hereafter is that the characteristic speeds inside and outside the tube satisfy the ordering for "photospheric conditions" in the sense of Figure 3 of Edwin and Roberts (1983). In particular, $\beta$ inside the tube is assumed to be smaller than unity. This assumption on the internal plasma $\beta$ is generally accepted for photospheric BPs, even though $\beta$ is difficult to measure directly (e.g. Shelyag et al., 2010; Cho et al., 2019).

Based on ideal MHD equations, setting the radial-velocity perturbation $v_{r}=A(r) \cos (k z-\omega t$ ), we can obtain (Freij et al., 2016, without considering azimuthal components of the velocity perturbance and magnetic field):

$$
\begin{gathered}
b_{z}=\frac{B_{0}}{\omega} \frac{1}{r} \frac{\partial(r A(r))}{\partial r} \sin (k z-\omega t), \\
p_{1}=c_{\mathrm{s}}^{2} \rho_{1}=-\frac{\omega \rho_{0} c_{\mathrm{s}}^{2}}{\left(c_{\mathrm{s}}^{2} k^{2}-\omega^{2}\right)} \frac{1}{r} \frac{\partial(r A(r))}{\partial r} \sin (k z-\omega t),
\end{gathered}
$$

where $b_{z}$ is the longitudinal magnetic-field perturbation, $B_{0}$ and $\rho_{0}$ are background magnetic field and density, $p_{1}$ is the pressure perturbation, and $\rho_{1}$ is the density perturbation.

Comparing Equations 1 and 2, we can state that if $c_{\mathrm{s}}^{2} k^{2}-\omega^{2}>0$ (i.e. the phase speed $c_{\mathrm{p}}=\omega / k$ is smaller than the sound speed $c_{\mathrm{s}}$, corresponding to slow sausage mode), then $b_{z}$ and $\rho_{1}$ (or $p_{1}$ ) will be $180^{\circ}$ out of phase (i.e. in antiphase). On the contrary, if $c_{\mathrm{s}}^{2} k^{2}-\omega^{2}<0$ (i.e. the phase speed is larger than the sound speed, corresponding to the fast sausage mode), then the perturbations will be in phase.

Since the magnetic flux in a flux tube is constant, we have $B_{0} S_{0}=\left(B_{0}+\right.$ $\left.b_{z}\right)\left(S_{0}-S_{1}\right)$. Here, $S_{0}$ and $S_{1}$ represent the unperturbed and perturbed crosssection areas, respectively. Ignoring $b_{z} S_{1}$, we can obtain $B_{0} S_{1}=-b_{z} S_{0}$, or

$$
\frac{S_{1}}{S_{0}}=-\frac{b_{z}}{B_{0}} .
$$

So the area perturbation $S_{1}$ and the longitudinal field perturbation $b_{z}$ are in anti-phase, and we can find the change of the magnetic field from the change of the area. This was also used by Grant et al. (2015).

As a result, for the slow sausage mode, fluctuations of area and density are in phase; whereas for the fast sausage mode, the fluctuations are in anti-phase. These simple phase relations have also been comfirmed by other theoretical 


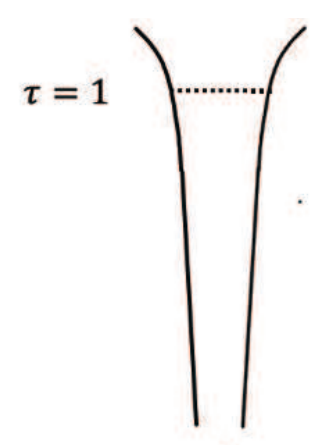

$t_{1}$

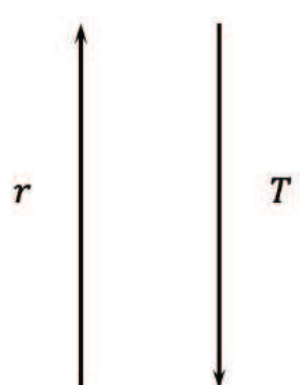

$T$

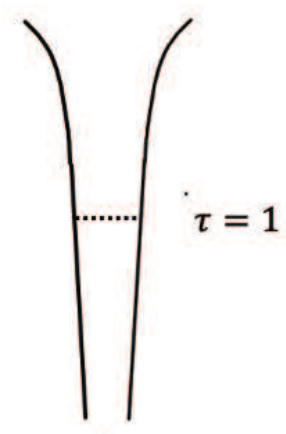

$t_{2}$

Figure 6. The change of intensity and area caused by the opacity effect in a flux tube. The dashed lines represent the $\tau=1$ surface, corresponding to the BP that we see in the TiO image. From time $t_{1}$ to $t_{2}$, the surface moves to a lower height. The two arrows in the middle indicate that the height increases from the bottom up and temperature increases from the top down.

investigations (Moreels, Goossens, and Van Doorsselaere, 2013; Moreels and Van Doorsselaere, 2013).

Next we will discuss the impact of the opacity effect and associate density with the continuum intensity, so that we can obtain the phase relation between fluctuations of intensity and area for fast and slow sausage modes.

\subsection{Phase Relation between Fluctuations of Intensity and Area}

The $\mathrm{TiO}$ data used in our study could be regarded as continuum images (see, e.g. LeBlanc, 2010), so the intensity that we observed could be written as

$$
I=\int_{0}^{\tau} \frac{\sigma T(\tau)^{4}}{\pi} \mathrm{e}^{-\tau} \mathrm{d} \tau
$$

where $\sigma$ is the Stefan-Boltzmann constant, and $T(\tau)$ is the local temperature at optical depth of $\tau$. Thus the observed intensity fluctuations come primarily from two sources: the change of temperature and the change of optical depth. The latter depends on density and temperature along the line-of-sight, which is known as the opacity effect (Fujimura and Tsuneta, 2009).

If the oscillations of intensity and area in BPs are caused by the opacity effect, then the oscillations will be in anti-phase. In Figure 6, a BP is shown as a laterally expanding flux tube, and the layer of $\tau=1$ is marked with the dashed line. Suppose that from time $t_{1}$ to $t_{2}$, the $\tau=1$ layer moves to a lower height, resulting in a reduced area of the BP. In the deeper layer with a higher temperature, the intensity would increase, which means that the intensity and area should be in anti-phase. Clearly it is contrary to our observation.

Without considering the opacity effect, we can assume that the surfaces that we see are at the same height, as shown in Figure 7. Another assumption that we made is that the $\mathrm{BP}$ intensity increases from time $t_{1}$ to $t_{2}$, and it is caused by the increase of temperature. The higher temperature will lead to a 

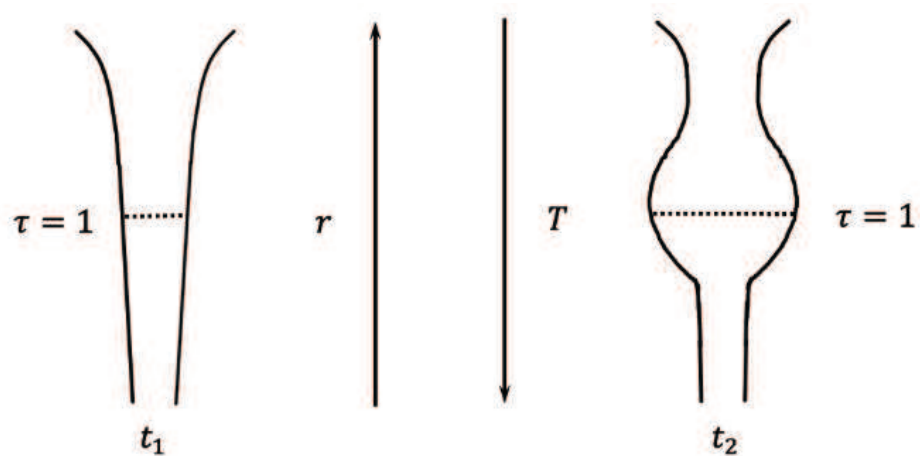

Figure 7. The change of intensity and area caused by the sausage waves. The $\tau=1$ surface remains the same height from time $t_{1}$ to $t_{2}$. The two arrows in the middle indicate that the height increases from the bottom up and temperature increases from the top down.

decrease in the absorption coefficient $\kappa_{\nu}$ at a frequency $\nu$, which is proportional to $\left[1-\exp \left(-\mathrm{h} \nu / \mathrm{k}_{\mathrm{B}} T\right)\right]$, where $\mathrm{h}$ and $\mathrm{k}_{\mathrm{B}}$ are the Planck constant and Boltzmann constant (Rutten, 1995). In order to ensure

$$
\tau=\int_{r_{0}}^{\infty} \kappa_{\nu} \rho(r) \mathrm{d} r=1,
$$

the density should increase. Here $\rho(r)$ represents the density at height of $r$, and $r_{0}$ represents height of the surface that we see. Our observation shows that the fluctuations of intensity and area are in phase. If the area increases at the same time, the density will be in phase with the area, which is a characteristic of the slow sausage wave (see Section 4.1). So the in-phase oscillations of intensity and area that we have observed could be possible evidence of slow sausage waves in BPs.

However, if we consider both the opacity effect and the presence of sausage modes, the result will be different. In Figure 8 , from $t_{1}$ to $t_{2}$, the sausage waves enlarge the cross-section area of the flux tube, and the magnetic field will decrease. If the opacity effect moves the $\tau=1$ layer to a lower height at the same time, we also expect in-phase oscillations of intensity and area, but now we cannot distinguish which mode the sausage waves are. The total pressure equilibrium at the same height is given by

$$
p_{\mathrm{i}}+\frac{B_{\mathrm{i}}^{2}}{2 \mu_{0}}=p_{\mathrm{e}}+\frac{B_{\mathrm{e}}^{2}}{2 \mu_{0}},
$$

where $p_{\mathrm{i}}$ and $p_{\mathrm{e}}$ are the internal and external thermal pressure, $B_{\mathrm{i}}$ and $B_{\mathrm{e}}$ are the internal and external magnetic field, and $\mu_{0}$ is the permeability. Equation 6 indicates that the decreasing magnetic field inside the tube will lead to an increasing thermal pressure, which is proportional to $\rho T$. If the density $\rho$ increases, the waves will be slow mode; otherwise they will be fast mode. For specific determination, it may be necessary to introduce some appropriate photospheric 


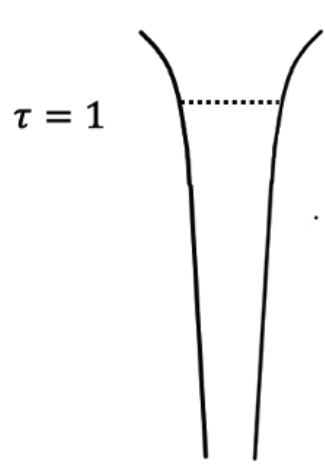

$t_{1}$
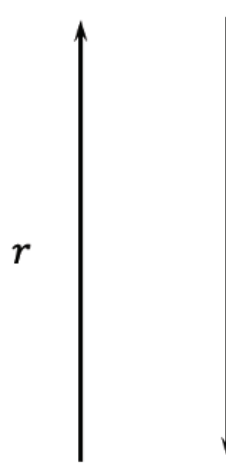

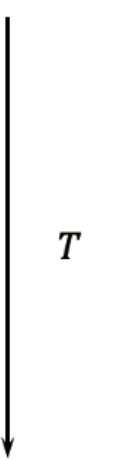

$T$

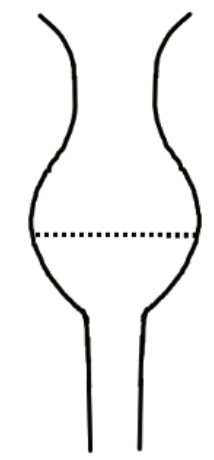

$t_{2}$

Figure 8. The changes of intensity and area caused by both the opacity effect and sausage waves. The $\tau=1$ surface moves to a lower height from time $t_{1}$ to $t_{2}$. The two arrows in the middle indicate that the height increases from the bottom up and temperature increases from the top down.

atmosphere models (e.g. Fang, Tang, and Xu, 2006), which will be our next step. Nevertheless, no matter which mode the waves actually are, the results that we observed can be seen as evidence for sausage waves in BPs.

\section{Conclusion and Remarks}

In this study, we used the $\mathrm{TiO}$ images obtained from the GST/BBSO on 6 August 2018 to carry out a detailed analysis of several isolated photospheric BPs. For the four selected BPs, the changes of their area and average intensity with time show obvious oscillations, and an in-phase relation.

In order to interpret these results, we presented a simple theoretical discussion, ruling out the opacity effect alone and identifying the observed oscillations in BPs as sausage modes. Assuming that the fluctuations are only caused by sausage waves, we can identify the oscillations in BPs as slow sausage modes, which have been found in some previous observations of magnetic pores (e.g. Grant et al., 2015; Freij et al., 2016). Our discovery provides strong support for the existence of sausage waves in the photospheric flux tube. Although the presence of sausage waves has also been observed in magnetic pores or sunspots, BPs are better tracers for the flux tubes, and they are more ubiquitous in the photosphere. Additionally, BPs are much smaller than pores and sunspots, which makes them more easily buffeted by surrounding granular motions. Since the dissipation of sausage modes is generally believed to have an important contribution to the heating of the chromospheric atmosphere (Grant et al., 2015; Yu, Van Doorsselaere, and Goossens, 2017; Chen et al., 2018; Keys et al., 2018; Gilchrist-Millar et al., 2021), our findings could be helpful for understanding the chromosphere heating.

Our future work will first focus on the mode identification by further addressing the opacity effect. We plan to introduce an appropriate photospheric 
atmosphere model to figure it out. Futhermore, our research is still limited to single-channel observations of the photosphere. We plan to combine more data in different wavelengths or spectral observations to study the propagation of sausage waves in BPs to the upper atmosphere. Moreover, the Daniel K. Inouye Solar Telescope (DKIST: Rast et al., 2021) can provide observations of the photosphere at an unprecedented high resolution. By using the oncoming data from DKIST, we may find more evidence of MHD waves in BPs and carry out more detailed study.

Acknowledgments We would like to thank Richard Morton, Mingde Ding, and Tom Van Doorsselaere for helpful comments and suggestions. This work has been supported by the Strategic Priority Research Program of CAS (grant XDA17040507) and NSFC grants 11825301 and 11790304. B. Li was supported by the NSFC grants 11761141002 and 41974200 . BBSO operation is supported by NJIT and US NSF AGS-1821294 grant. GST operation is partly supported by the Korea Astronomy and Space Science Institute, the Seoul National University, and the Key Laboratory of Solar Activities of Chinese Academy of Sciences (CAS) and the Operation, Maintenance and Upgrading Fund of CAS for Astronomical Telescopes and Facility Instruments.

Disclosure of Potential Conflicts of Interest The authors declare that they have no conflicts of interest.

\section{References}

Bellot Rubio, L.R., Rodríguez Hidalgo, I., Collados, M., Khomenko, E., Ruiz Cobo, B.: 2001, Observation of Convective Collapse and Upward-moving Shocks in the Quiet Sun. Astrophys. J. 560, 1010. DOI. ADS.

Berger, T.E., Title, A.M.: 1996, On the Dynamics of Small-Scale Solar Magnetic Elements. Astrophys. J. 463, 365. DOI. ADS.

Berger, T.E., Schrijver, C.J., Shine, R.A., Tarbell, T.D., Title, A.M., Scharmer, G.: 1995, New Observations of Subarcsecond Photospheric Bright Points. Astrophys. J. 454, 531. DOI. ADS.

Berger, T.E., Löfdahl, M.G., Shine, R.A., Title, A.M.: 1998, Measurements of Solar Magnetic Element Dispersal. Astrophys. J. 506, 439. DOI. ADS.

Bovelet, B., Wiehr, E.: 2003, Dynamics of the solar active region finestructure. Astron. Astrophys. 412, 249. DOI. ADS.

Cao, W., Gorceix, N., Coulter, R., Ahn, K., Rimmele, T.R., Goode, P.R.: 2010, Scientific instrumentation for the $1.6 \mathrm{~m}$ New Solar Telescope in Big Bear. Astronomische Nachrichten 331, 636. DOI. ADS.

Chen, S.-X., Li, B., Xiong, M., Yu, H., Guo, M.-Z.: 2015, Standing Sausage Modes in Nonuniform Magnetic Tubes: An Inversion Scheme for Inferring Flare Loop Parameters. Astrophys. J. 812, 22. DOI. ADS.

Chen, S.-X., Li, B., Shi, M., Yu, H.: 2018, Damping of Slow Surface Sausage Modes in Photospheric Waveguides. Astrophys. J. 868, 5. DOI. ADS.

Cho, I.-H., Moon, Y.-J., Nakariakov, V.M., Yu, D.J., Lee, J.-Y., Bong, S.-C., Kim, R.-S., Cho, K.-S., Kim, Y.-H., Lee, J.-O.: 2019, Seismological Determination of the Alfvén Speed and Plasma Beta in Solar Photospheric Bright Points. Astrophys. J. Lett. 871, L14. DOI. ADS.

De Pontieu, B.: 2002, High-Resolution Observations of Small-Scale Emerging Flux in the Photosphere. Astrophys. J. 569, 474. DOI. ADS.

Defouw, R.J.: 1976, Wave propagation along a magnetic tube. Astrophys. J. 209, 266. DOI. ADS.

Dorotovič, I., Erdélyi, R., Karlovský, V.: 2008, Identification of linear slow sausage waves in magnetic pores. 247, 351. DOI. ADS. 
Dunn, R.B., Zirker, J.B.: 1973, The Solar Filigree. Solar Phys. 33, 281. DOI. ADS.

Edwin, P.M., Roberts, B.: 1983, Wave Propagation in a Magnetic Cylinder. Solar Phys. 88, 179. DOI. ADS.

Fang, C., Tang, Y.-H., Xu, Z.: 2006, Spectral Analysis and Atmospheric Models of Microflares. Chin. J. Astron. Astrophys. 6, 597. DOI. ADS.

Freij, N., Dorotovič, I., Morton, R.J., Ruderman, M.S., Karlovský, V., Erdélyi, R.: 2016, On the Properties of Slow MHD Sausage Waves within Small-scale Photospheric Magnetic Structures. Astrophys. J. 817, 44. DOI. ADS.

Fujimura, D., Tsuneta, S.: 2009, Properties of Magnetohydrodynamic Waves in the Solar Photosphere Obtained with Hinode. Astrophys. J. 702, 1443. DOI. ADS.

Gilchrist-Millar, C.A., Jess, D.B., Grant, S.D.T., Keys, P.H., Beck, C., Jafarzadeh, S., Riedl, J.M., Van Doorsselaere, T., Ruiz Cobo, B.: 2021, Magnetoacoustic wave energy dissipation in the atmosphere of solar pores. Mon. Not. Roy. Astron. Soc. 379, 20200172. DOI. ADS.

Grant, S.D.T., Jess, D.B., Moreels, M.G., Morton, R.J., Christian, D.J., Giagkiozis, I., Verth, G., Fedun, V., Keys, P.H., Van Doorsselaere, T., Erdélyi, R.: 2015, Wave Damping Observed in Upwardly Propagating Sausage-mode Oscillations Contained within a Magnetic Pore. Astrophys. J. 806, 132. DOI. ADS.

Guo, M.-Z., Chen, S.-X., Li, B., Xia, L.-D., Yu, H.: 2016, Inferring Flare Loop Parameters with Measurements of Standing Sausage Modes. Solar Phys. 291, 877. DOI. ADS.

Jafarzadeh, S., Solanki, S.K., Stangalini, M., Steiner, O., Cameron, R.H., Danilovic, S.: 2017, High-frequency Oscillations in Small Magnetic Elements Observed with Sunrise/SuFI. Astrophys. J. Suppl. 229, 10. DOI. ADS.

Jess, D.B., Mathioudakis, M., Erdélyi, R., Crockett, P.J., Keenan, F.P., Christian, D.J.: 2009, Alfvén Waves in the Lower Solar Atmosphere. Science 323, 1582. DOI. ADS.

Jess, D.B., Shelyag, S., Mathioudakis, M., Keys, P.H., Christian, D.J., Keenan, F.P.: 2012a, Propagating Wave Phenomena Detected in Observations and Simulations of the Lower Solar Atmosphere. Astrophys. J. 746, 183. DOI. ADS.

Jess, D.B., Pascoe, D.J., Christian, D.J., Mathioudakis, M., Keys, P.H., Keenan, F.P.: 2012b, The Origin of Type I Spicule Oscillations. Astrophys. J. Lett. 744, L5. DOI. ADS.

Keys, P.H., Mathioudakis, M., Jess, D.B., Shelyag, S., Crockett, P.J., Christian, D.J., Keenan, F.P.: 2011, The Velocity Distribution of Solar Photospheric Magnetic Bright Points. Astrophys. J. Lett. 740, L40. DOI. ADS.

Keys, P.H., Mathioudakis, M., Jess, D.B., Mackay, D.H., Keenan, F.P.: 2014, Dynamic properties of bright points in an active region. Astron. Astrophys. 566, A99. DOI. ADS.

Keys, P.H., Morton, R.J., Jess, D.B., Verth, G., Grant, S.D.T., Mathioudakis, M., Mackay, D.H., Doyle, J.G., Christian, D.J., Keenan, F.P., Erdélyi, R.: 2018, Photospheric Observations of Surface and Body Modes in Solar Magnetic Pores. Astrophys. J. 857, 28. DOI. ADS.

Keys, P.H., Reid, A., Mathioudakis, M., Shelyag, S., Henriques, V.M.J., Hewitt, R.L., Del Moro, D., Jafarzadeh, S., Jess, D.B., Stangalini, M.: 2020, High-resolution spectropolarimetric observations of the temporal evolution of magnetic fields in photospheric bright points. Astron. Astrophys. 633, A60. DOI. ADS.

Kolotkov, D.Y., Smirnova, V.V., Strekalova, P.V., Riehokainen, A., Nakariakov, V.M.: 2017, Long-period quasi-periodic oscillations of a small-scale magnetic structure on the Sun. Astron. Astrophys. 598, L2. DOI. ADS.

Kuckein, C.: 2019, Height variation of magnetic field and plasma flows in isolated bright points. Astron. Astrophys. 630, A139. DOI. ADS.

LeBlanc, F.: 2010, An Introduction to Stellar Astrophysics, John Wiley \& Sons, Chichester, UK. ADS.

Li, B., Antolin, P., Guo, M.-Z., Kuznetsov, A.A., Pascoe, D.J., Van Doorsselaere, T., Vasheghani Farahani, S.: 2020, Magnetohydrodynamic Fast Sausage Waves in the Solar Corona. Space Sci. Rev. 216, 136. DOI. ADS.

Liu, Y., Xiang, Y., Erdélyi, R., Liu, Z., Li, D., Ning, Z., Bi, Y., Wu, N., Lin, J.: 2018, Studies of Isolated and Non-isolated Photospheric Bright Points in an Active Region Observed by the New Vacuum Solar Telescope. Astrophys. J. 856, 17. DOI. ADS.

Martínez González, M.J., Asensio Ramos, A., Manso Sainz, R., Khomenko, E., Martínez Pillet, V., Solanki, S.K., López Ariste, A., Schmidt, W., Barthol, P., Gandorfer, A.: 2011, Unnoticed Magnetic Field Oscillations in the Very Quiet Sun Revealed by SUNRISE/IMaX. Astrophys. J. Lett. 730, L37. DOI. ADS.

Mehltretter, J.P.: 1974, Observations of Photospheric Faculae at the Center of the Solar Disk. Solar Phys. 38, 43. DOI. ADS. 
Moreels, M.G., Van Doorsselaere, T.: 2013, Phase relations for seismology of photospheric flux tubes. Astron. Astrophys. 551, A137. DOI. ADS.

Moreels, M.G., Goossens, M., Van Doorsselaere, T.: 2013, Cross-sectional area and intensity variations of sausage modes. Astron. Astrophys. 555, A75. DOI. ADS.

Morton, R.J., Erdélyi, R., Jess, D.B., Mathioudakis, M.: 2011, Observations of Sausage Modes in Magnetic Pores. Astrophys. J. Lett. 729, L18. DOI. ADS.

Morton, R.J., Verth, G., Jess, D.B., Kuridze, D., Ruderman, M.S., Mathioudakis, M., Erdélyi, R.: 2012, Observations of ubiquitous compressive waves in the Sun's chromosphere. Nature Communications 3, 1315. DOI. ADS.

Mumford, S.J., Fedun, V., Erdélyi, R.: 2015, Generation of Magnetohydrodynamic Waves in Low Solar Atmospheric Flux Tubes by Photospheric Motions. Astrophys. J. 799, 6. DOI. ADS.

Nagata, S., Tsuneta, S., Suematsu, Y., Ichimoto, K., Katsukawa, Y., Shimizu, T., Yokoyama, T., Tarbell, T.D., Lites, B.W., Shine, R.A., Berger, T.E., Title, A.M., Bellot Rubio, L.R., Orozco Suárez, D.: 2008, Formation of Solar Magnetic Flux Tubes with Kilogauss Field Strength Induced by Convective Instability. Astrophys. J. Lett. 677, L145. DOI. ADS.

Nakariakov, V.M., Melnikov, V.F., Reznikova, V.E.: 2003, Global sausage modes of coronal loops. Astron. Astrophys. 412, L7. DOI. ADS.

Rast, M.P., Bello González, N., Bellot Rubio, L., Cao, W., Cauzzi, G., Deluca, E., de Pontieu, B., Fletcher, L., Gibson, S.E., Judge, P.G., Katsukawa, Y., Kazachenko, M.D., Khomenko, E., Landi, E., Martínez Pillet, V., Petrie, G.J.D., Qiu, J., Rachmeler, L.A., Rempel, M., Schmidt, W., Scullion, E., Sun, X., Welsch, B.T., Andretta, V., Antolin, P., Ayres, T.R., Balasubramaniam, K.S., Ballai, I., Berger, T.E., Bradshaw, S.J., Campbell, R.J., Carlsson, M., Casini, R., Centeno, R., Cranmer, S.R., Criscuoli, S., Deforest, C., Deng, Y., Erdélyi, R., Fedun, V., Fischer, C.E., González Manrique, S.J., Hahn, M., Harra, L., Henriques, V.M.J., Hurlburt, N.E., Jaeggli, S., Jafarzadeh, S., Jain, R., Jefferies, S.M., Keys, P.H., Kowalski, A.F., Kuckein, C., Kuhn, J.R., Kuridze, D., Liu, J., Liu, W., Longcope, D., Mathioudakis, M., McAteer, R.T.J., McIntosh, S.W., McKenzie, D.E., Miralles, M.P., Morton, R.J., Muglach, K., Nelson, C.J., Panesar, N.K., Parenti, S., Parnell, C.E., Poduval, B., Reardon, K.P., Reep, J.W., Schad, T.A., Schmit, D., Sharma, R., Socas-Navarro, H., Srivastava, A.K., Sterling, A.C., Suematsu, Y., Tarr, L.A., Tiwari, S., Tritschler, A., Verth, G., Vourlidas, A., Wang, H., Wang, Y.-M., NSO and DKIST Project, DKIST Instrument Scientists, DKIST Science Working Group, DKIST Critical Science Plan Community: 2021, Critical Science Plan for the Daniel K. Inouye Solar Telescope (DKIST). Solar Phys. 296, 70. DOI. ADS.

Roberts, B.: 2019, MHD waves in the solar atmosphere, Cambridge University Press, Cambridge, UK.

Rutten, R.J.: 1995, Radiative transfer in stellar atmospheres, Sterrekundig Instituut Utrecht.

Sánchez Almeida, J., Márquez, I., Bonet, J.A., Domínguez Cerdeña, I., Muller, R.: 2004, Bright Points in the Internetwork Quiet Sun. Astrophys. J. Lett. 609, L91. DOI. ADS.

Shelyag, S., Mathioudakis, M., Keenan, F.P., Jess, D.B.: 2010, A photospheric bright point model. Astron. Astrophys. 515, A107. DOI. ADS.

Solanki, S.K., Zufferey, D., Lin, H., Rueedi, I., Kuhn, J.R.: 1996, Infrared lines as probes of solar magnetic features. XII. Magnetic flux tubes: evidence of convective collapse? Astron. Astrophys. 310, L33. ADS.

Spruit, H.C.: 1979, Convective collapse of flux tubes. Solar Phys. 61, 363. DOI. ADS.

Stangalini, M., Berrilli, F., Consolini, G.: 2013, The spectrum of kink-like oscillations of solar photospheric magnetic elements. Astron. Astrophys. 559, A88. DOI. ADS.

Stangalini, M., Giannattasio, F., Jafarzadeh, S.: 2015, Non-linear propagation of kink waves to the solar chromosphere. Astron. Astrophys. 577, A17. DOI. ADS.

Stangalini, M., Solanki, S.K., Cameron, R., Martínez Pillet, V.: 2013, First evidence of interaction between longitudinal and transverse waves in solar magnetic elements. Astron. Astrophys. 554, A115. DOI. ADS.

Tian, H., Young, P.R., Reeves, K.K., Wang, T., Antolin, P., Chen, B., He, J.: 2016, Global Sausage Oscillation of Solar Flare Loops Detected by the Interface Region Imaging Spectrograph. Astrophys. J. Lett. 823, L16. DOI. ADS.

Utz, D., Hanslmeier, A., Muller, R., Veronig, A., Rybák, J., Muthsam, H.: 2010, Dynamics of isolated magnetic bright points derived from Hinode/SOT G-band observations. Astron. Astrophys. 511, A39. DOI. ADS. 
Van Doorsselaere, T., De Groof, A., Zender, J., Berghmans, D., Goossens, M.: 2011, LYRA Observations of Two Oscillation Modes in a Single Flare. Astrophys. J. 740, 90. DOI. ADS.

Yu, D.J., Van Doorsselaere, T., Goossens, M.: 2017, Resonant absorption of the slow sausage wave in the slow continuum. Astron. Astrophys. 602, A108. DOI. ADS.

Zimovets, I.V., McLaughlin, J.A., Srivastava, A.K., Kolotkov, D.Y., Kuznetsov, A.A., Kupriyanova, E.G., Cho, I.-H., Inglis, A.R., Reale, F., Pascoe, D.J., Tian, H., Yuan, D., Li, D., Zhang, Q.M.: 2021, Quasi-Periodic Pulsations in Solar and Stellar Flares: A Review of Underpinning Physical Mechanisms and Their Predicted Observational Signatures. Space Sci. Rev. 217, 66. DOI. ADS. 